\title{
FATAL CASE OF WASP STINGING
}

\section{Senanayake S.M.H.M.K. \& Senarathna K.N.}

Teaching Hospital, Anuradhapura, Sri Lanka

Corresponding Author: Senanayake S.M.H.M.K.

\begin{abstract}
History - While a 2 year and 9 month old girl was going for a bath with the grandmother, they encountered a wasp net on the ground. The wasps stung the child. She developed anaphylaxis and died during transport from local hospital eight hours after the attack.

Postmortem examination- Thirty two skin lesions of 2-3 mm wide, circular, black macules were seen on back and limbs. Every black spot was surrounded with $0.5-2 \mathrm{~cm}$ size elevated red margin. Pink fluid in pleural cavities and peritoneal cavity, shock lungs and congested viscera were the only internal findings. Blood was hemolyzed and unsuitable for serum beta tryptase test. Based on the skin lesion, medical history and evidence of eye witness, the cause of death was pronounced as anaphylaxis following wasp attack.

Conclusion- Familiarization with skin lesions of wasp stings are important for Srilankan forensic medicine practitioners. Collection and preservation of ante mortem blood samples or early postmortem blood sample with serum separation by centrifuging is advisable in suspected anaphylaxis cases for future confirmatory investigation.
\end{abstract}

Key words: Vespa affinis, skin lesion, wasp sting, anaphylaxis, envenomation 


\section{INTRODUCTION}

In the animal kingdom, order Hymenoptera and suborder Apocrita includes bees, yellow jackets, hornets (wasps) and ants. ${ }^{1}$ Wasps can be divided into social wasps and solitary wasps. Social wasps are aggressive and live as colonies in nests found in tree stumps, on rocks surfaces, tall structures like buildings, roof of caves, and mammal burrows. More than 25000 species of wasps exist in the world and cause more morbidity and mortality than other venomous animals. $^{2} \quad$ In Sri Lanka, Vespa affinis (debara) species is mainly responsible for the highest number of deaths among Hymenoptera stings. ${ }^{3,4}$

The stinging apparatus is a modified ovipositor, hence the sting ability belongs to females. ${ }^{3}$ Stinging apparatus is connected to a venom gland and used as an offensive or defensive weapon. Unlike bees, wasps can sting multiple times. ${ }^{5}$ The venom of wasp contains a mixture of Histamine releasing factors, enzymes, hemolysins, neurotoxins, vasodilators, vasospastic amines and Phospholipase A. Hornet stinging is a common environmental hazard in Sri Lanka. When disturbed, hornets attack in swarm. Majority of victims recover in a hospitals but some die of complications.

Clinical features of hornet stings are localized redness, swelling, and pain over the site of the wasp sting. The pain begins immediately, and gradually worsens as the redness and edema gradually worsen. The localized reaction may last 6-12 hours. A minute punctate lesion is visible at the center of the lesion where the wasp sting occurred. Patients with a mild allergic reaction may experience itching and hives. Patients with severe allergic reactions and anaphylaxis may present with throatclosing sensation, dyspnoea, chest tightness, lightheadedness, increased anxiety, headache, nausea, abdominal cramps, palpitations, symptoms of Myasthenia gravis and optic neuropathy. ${ }^{6}$ Death due to anaphylaxis can occur following single sting. ${ }^{7}$ Envenomation occurs due to more than 20 stings and death is due to hemolysis, rhabdomyolysis and acute kidney injury. ${ }^{8}$ Disseminated intravascular coagulation and allergic myocardial infarctions (kounis syndrome) are the late fatal complications. ${ }^{9}$

A range of clinical complications ranging from nonspecific skin lesions to anaphylactic shock can occur, in a previously sensitized person, following even a single hymenopteran bite (Vespa affinis). ${ }^{10}$

When there is a death, external examination for wasp stings, internal examination for organ failure and blood investigations for mast cell tryptase and Ig E levels will help the diagnosis. We report a fatal case of wasp sting with 32 skin lesions.

\section{CASE REPORT}

History- A two years and nine month old girl had approached a fallen wasp net on the ground. A swarm of wasps had attacked the girl and grandmother at about $2.30 \mathrm{pm}$. At $6.30 \mathrm{pm}$ girl was transferred to tertiary care hospital from local hospital. Patient died at $8.40 \mathrm{pm}$ on admission to the intensive care unit.

Postmortem examination- thirty two skin lesions were seen on limbs and back. Each lesion was a black circular macule $2-3 \mathrm{~mm}$ in size, surrounded by an elevated erythematous margin of 0.5 to $2 \mathrm{~cm}$ in size. Internally, bilateral pleural effusion, ascitis, dark rubbery lungs and pale kidneys with unclear cortico-medullary junctions were seen. Histology of skin showed only ectatic blood vessels. 


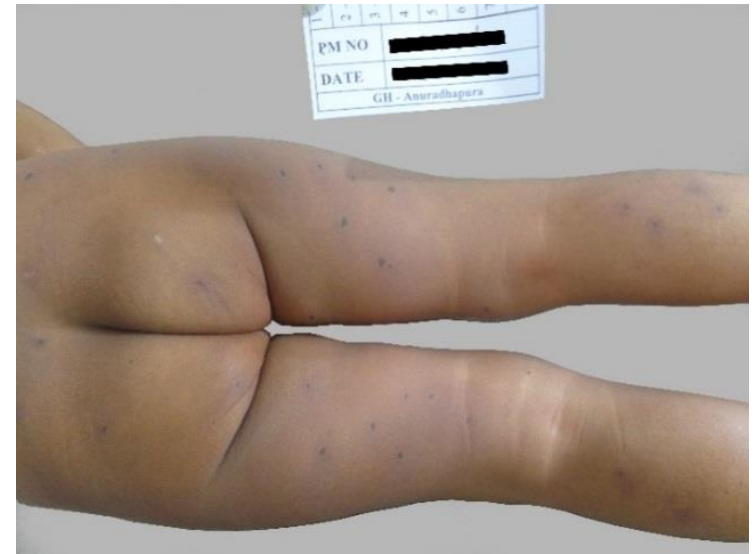

Fig. 1 : Skin lesions on legs

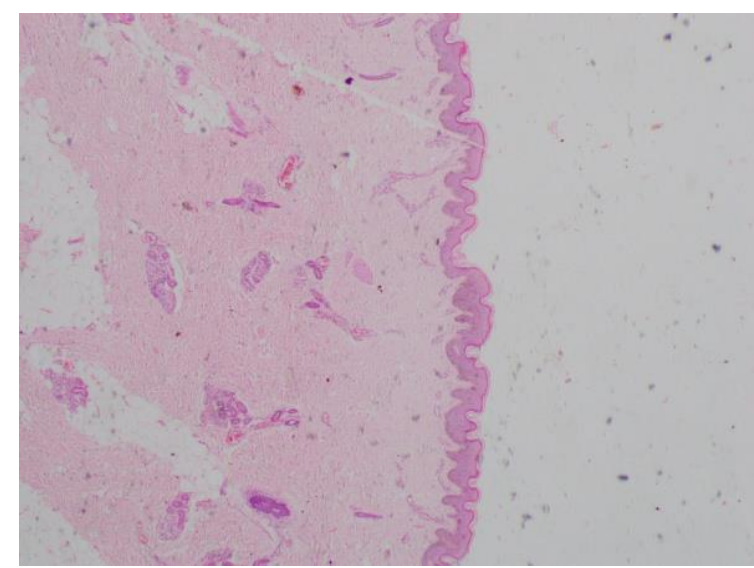

Fig. 3: Photomicrograph of (x4)

Section of skin with ectatic blood vessels (Hematoxylin and eosin stain)

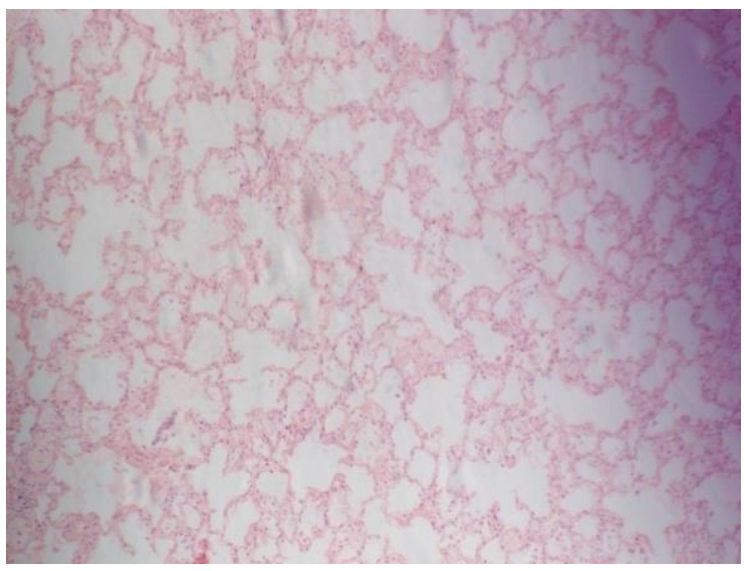

Fig. 5: Photomicrograph of Lung (x10) Section of lung shows no pulmonary edema evidence or micro thrombi (Hematoxylin and eosin stain)

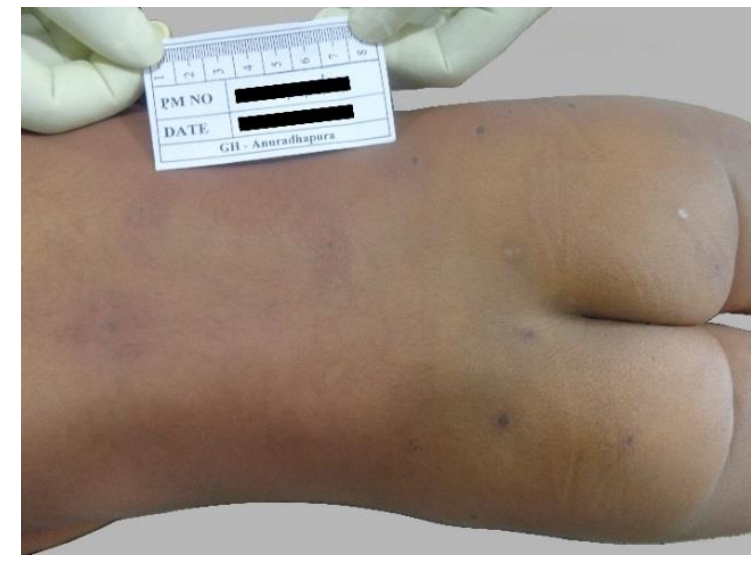

Fig. 2 : Skin lesions on back

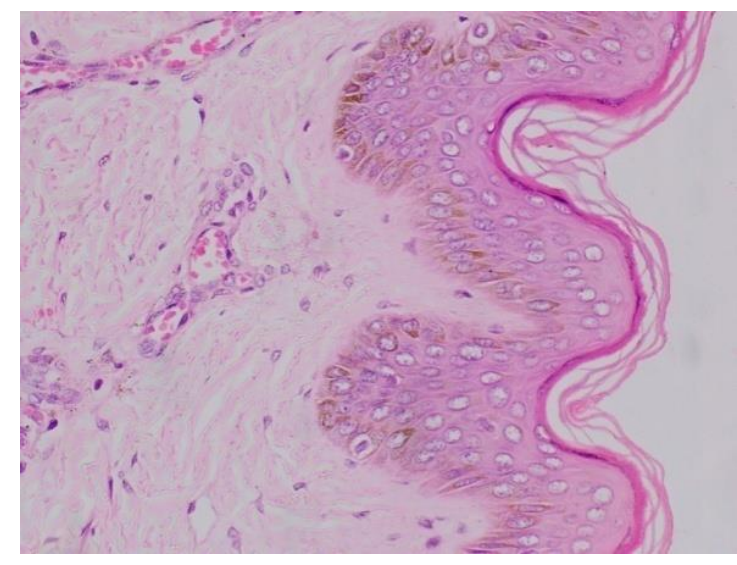

Fig. 4: Photomicrograph of $(\mathrm{x} 40)$ Section of skin with no increase complement of inflammatory sells (Hematoxylin and eosin stain)

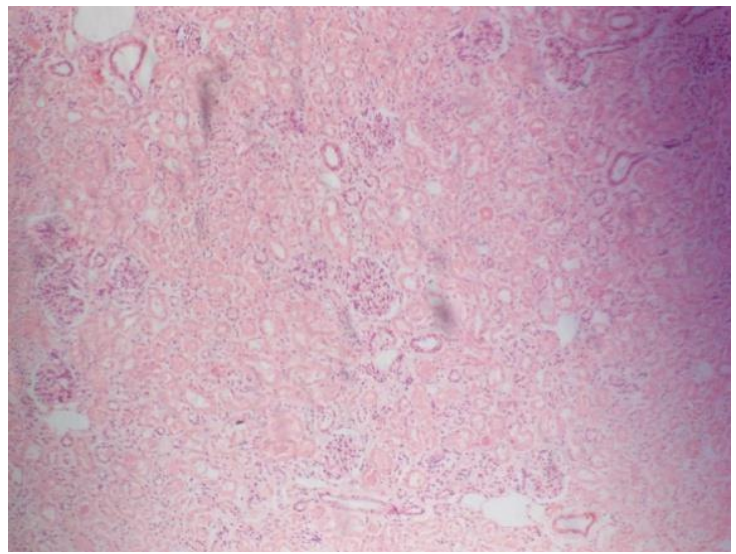

Fig. 6: Photomicrograph of Kidney Section of kidney show no of acute tubular necrosis (Hematoxylin and eosin stain) 


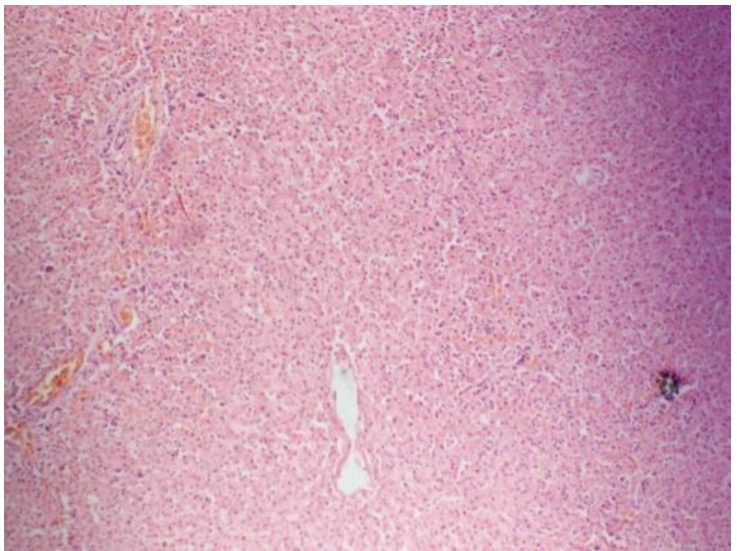

Fig. 7: Photomicrograph of Liver (x10) Section of liver show Congestion of Blood vessel with no evidence of micro thrombi (Hematoxylin and eosin stain)

Blood was hemolysed and unsuitable for beta trypatse investigation. After exclusion of trauma and natural diseases, with the help of eye witness and medical records of local hospital, the cause of death was pronounced as anaphylaxis following wasp stings.

\section{DISCUSSION}

This is a witnessed case of wasp sting and the diagnosis was obvious for the clinicians and forensic pathologists. Because wasps are abundant in Sri Lanka and nesting close to human dwelling, stings are frequently reported on unsuspecting children and adults. Other than the history the most important clinical feature is the skin lesion. Punctuate dark color circular centre (1$2 \mathrm{~mm}$ ) with raised red circular margin (1$2 \mathrm{~cm}$ ) was observed in all 32 lesions of this case. The skin lesion was the only helpful clinical criterion for the diagnosis of wasp sting. Familiarization of skin lesion of wasp sting is important for the forensic practitioner to medically establish the diagnosis in both, witnessed deaths and also in the investigations of unwitnessed deaths recovered from jungles to exclude the possibility of wasp sting. Internal examination will be helpful to establish immediate cause of deaths such as multi organ failure due to anaphylaxis, acute pulmonary oedema, cardiotoxicity and acute kidney injury due to envenomation. ${ }^{6}$ Histology of skin lesions usually shows ectatic blood vessels and inflammatory cell infiltration. Absence of inflammatory cells in this case may be due to early death before cell migration.

To confirm anaphylaxis following wasp sting needs blood investigations but hemolysis would be an obstacle as in this case. Therefore preservation of antemortem blood sample and early collection of postmortem blood samples should be a routine practice in all suspected deaths due to anaphylaxis. Failure of the medical management in this case may be due to acuteness and severity of the allergic reaction as well as the younger age.

\section{CONCLUSION}

Familiarization of skin lesions of wasp sting is important for forensic practitioners. Preservation of antemortem blood sample and early collection of postmortem serum sample after centrifuging blood should be a routine practice in all suspected deaths due to anaphylaxis. 


\section{REFERENCES}

1. Akre RD, Reed HC: Ants, wasps and bees. Medical and veterinary Entomology. Edited by: Garry M, Lance D. 2002, London: Academic Press, 383-408.

2. Goulet H, Huber JT: Hymenoptera of the World: An Identification Guide to Families. 1993, Ottawa: Canada Communication Group.

3. Kularatne SAM, Gawarammana IB, De Silva PHJG: Severe multi-organ dydfunction following multiple wasp (Vespa affinis|) Stings. CMJ. 2003, 48: 146-147.

4. Kularathne K,Kannaagara T,Jayasena A,etal. Fatal acute pulmonary oedema and acute renal failure following wasp/hornetstinge in Sri Lanka: two case reports. Journal of medical case reports.2014 June13.

https://www.researchgate.net/publication/2 63100451_Fatal_acute_pulmonary_oedem a_and_acute_renal_failure_following_mul tiple_wasphornet_Vespa_affinis_stings_in _Sri_Lanka_Two_case_reports
5. Pick T: Wasp kinins and kinin analogues. Animal Toxins; Facts and Protocols. Edited by: Rochat H, Martin MF. 2000, Basel: Birkhauser, 100-103.

6. Maltzman JS, Lee AG, Miller NR: Optic neuropathy occurring after bee and wasp sting. Ophthalmology 2000, 107(1):193195.

7. Sharmila R, Chetan G, Narayan $P$, Srinivasan S.Multiorgan dysfunction syndrome following single wasp sting. The Indian Journal of paediatrics. $2007 \mathrm{Dec}$; 74(12) 1111-2

8. Vettr RS, Visscher PK, Camzine S. Mass envenomation by honey bees and wasps. Western Journal of Medicine. 1999 Apr; 170(4): 223-227 ( PMCID: PMC1305553)

9. George P, Pawar B, Calton N, Mathew P. Wasp sting: An unusual fatal outcome. Saudi Journal of kidney diseases and transplantation. 2008; 19(6) 969-972

10. Gunasekara WDVN, Ratnatunga NVI, Abeygunawardana AS: Acute renal failure following multiple wasp bites. Sri Lanka J Child Health 2007,36:67-68.13. 